\title{
A short note on Dynamic Stabilization of Moving Particle Semi-implicit method
}

\section{$\operatorname{AUTHOR}(\mathrm{S})$ :}

Tsuruta, Naoki; Khayyer, Abbas; Gotoh, Hitoshi

\section{CITATION:}

Tsuruta, Naoki ... [et al]. A short note on Dynamic Stabilization of Moving Particle Semi-implicit method. Computers \& Fluids 2013, 82: 158-164

\section{ISSUE DATE:}

2013-08

URL:

http://hdl.handle.net/2433/175511

\section{RIGHT:}

(C) 2013 Elsevier Ltd.; This is not the published version. Please cite only the published version.; この論文は出版社版でありません。引用の際に は出版社版をご確認ご利用ください。 


\section{Highlights}

A dynamically stabilized Moving Particle Semi-implicit method is developed.

A new scheme is proposed to provide meticulously adequate interparticle repulsive forces. The new scheme is shown to provide physically sound and computationally stable results. Verifications with different applications are performed to show the stabilizations. 


\title{
A Short Note on Dynamic Stabilization of Moving Particle Semi-implicit Method
}

\author{
Naoki Tsuruta ${ }^{1}$, Abbas Khayyer ${ }^{2}$, Hitoshi Gotoh ${ }^{3}$ \\ Department of Civil and Earth Resources Engineering, Kyoto University, \\ Katsura Campus, Nishikyo-ku, Kyoto 615-8540, Japan
}

This technical note presents a simple and effective scheme for Dynamic Stabilization of MPS method. The new scheme, abbreviated as DS, reproduces meticulously adequate repulsive forces to attenuate the interparticle penetration and thus stabilizes the calculations, even for highly deformed flows characterized by tensile stress states. By performing a set of simple two-phase flow simulations, we also show the inappropriateness of the simplified/anti-symmetric MPS pressure gradient models as they may result in predominant excessive repulsive forces and thus being unable to simulate the main flow features. The DS scheme is shown to provide physically sound and computationally stable simulations of such flows.

Keywords: MPS method, dynamic stabilization, particle method, tensile instability, multiphase flows

\section{Introduction}

The MPS (Moving Particle Semi-implicit) method was originally proposed by Koshizuka and Oka [1] for viscous incompressible flow as a Lagrangian mesh-free method. Lagrangian approach in hydraulic flow simulation is free from the non-linear advection term causing numerical diffusion, however, it simultaneously may result in instability due to the maldistribution of calculation points (particles). Up to now, the instability issue, as a general problem of particle method, has been widely studied [2-9]. In MPS-based simulations, the main causes of the maldistribution of particles correspond to either underestimation of interparticle repulsive forces or overestimation of interparticle attractive forces.

Due to the predominancy of attractive force overestimation, Koshizuka et al. [10] modified the original MPS gradient model so that the pressure interacting forces would be purely repulsive, and some

\footnotetext{
1 Corresponding Author, TEL: +81-75-383-3312 ; FAX: +81-75-383-3311 ; Email: tsuruta.naoki.57v@ st.kyoto-u.ac.jp

2 Email: khayyer@particle.kuciv.kyoto-u.ac.jp

3 Email: gotoh@particle.kuciv.kyoto-u.ac.jp
} 
so-far proposed modifications including the so-called CMPS method [11] are based on this concept. This approach is generally composed of an artificial repulsive force which vanishes for a perfectly symmetric distribution of neighboring particles. As a result, stability and accuracy would depend on the instantaneous distribution of calculation points. The concept of repulsive interparticle forces for enhancement of stability has also been introduced in the SPH framework [4] by incorporating a stress-dependent repulsive force term multiplied by a constant coefficient.

To minimize the tensile instability issue in MPS framework, Khayyer and Gotoh [8] proposed a Gradient Correction (GC) scheme. Nevertheless, this scheme does not resolve the maldistribution of particles and requires a meticulous setting of calculation condition with prudence. In a recent paper, Khayyer and Gotoh [9] showed that multiphase particle-based simulations are prone to become easily destabilized as a result of unphysical perturbations in particle motion and resulting interparticle penetrations.

In brief, a stabilization approach regardless of stress state is required for stable and accurate particle-based simulation. This short note highlights the particle method related to instability and the unphysical states of so-far proposed MPS methods stabilized by repulsive forces. A modification, namely a Dynamically Stabilized scheme, is proposed to resolve these problems. The new scheme provides meticulously adequate repulsive forces based on the Newton's third law of motion for both compressive and tensile stress states. It is shown to stabilize and adjust the disorder of calculation points for comprehensive MPS applications.

\section{The gradient model and instability}

In this section, previous and present gradient terms in the MPS method are explained. Detailed descriptions of other differential operators of standard and improved MPS methods can be referred to [1, $8,12]$.

In MPS method, the gradient operator for the pressure gradient [10] is defined as:

$$
\langle\nabla p\rangle_{i}=\frac{D_{s}}{n_{0}} \sum_{j \neq i} \frac{p_{j}-\hat{p}_{i}}{\left|\boldsymbol{r}_{j}-\boldsymbol{r}_{i}\right|^{2}}\left(\boldsymbol{r}_{j}-\boldsymbol{r}_{i}\right) w\left(\left|\boldsymbol{r}_{j}-\boldsymbol{r}_{i}\right|\right)
$$




$$
\hat{p}_{i}=\min _{j \subset J}\left(p_{i}, p_{j}\right), J=\left\{j: w\left(\left|\boldsymbol{r}_{j}-\boldsymbol{r}_{i}\right|\right) \neq 0\right\}
$$

where $p$ is the pressure, $D_{s}$ is number of space dimensions, $\boldsymbol{r}$ is coordinate vector, $w(r)$ is the kernel function and $n_{0}$ is the reference particle number density. The subscripts $i$ and $j$ correspond to target particle and its neighboring particles, respectively.

The Corrected MPS (CMPS) method [11] modifies Eq. 1 to enforce interparticle anti-symmetric interactions with consideration of the Newton's third law:

$$
\langle\nabla p\rangle_{i}=\frac{D_{s}}{n_{0}} \sum_{j \neq i} \frac{\left(p_{i}+p_{j}\right)-\left(\hat{p}_{i}+\hat{p}_{j}\right)}{\left|\boldsymbol{r}_{j}-\boldsymbol{r}_{i}\right|^{2}}\left(\boldsymbol{r}_{j}-\boldsymbol{r}_{i}\right) w\left(\left|\boldsymbol{r}_{j}-\boldsymbol{r}_{i}\right|\right)
$$

Considering the original gradient term [1] and the artificial repulsive force term for stabilization, Eq. 1 can be rewritten as:

$$
\langle\nabla p\rangle_{i}=\frac{D_{s}}{n_{0}} \sum_{j \neq i} \frac{\left(p_{j}-p_{i}\right)}{\left|\boldsymbol{r}_{j}-\boldsymbol{r}_{i}\right|^{2}}\left(\boldsymbol{r}_{j}-\boldsymbol{r}_{i}\right) w\left(\left|\boldsymbol{r}_{j}-\boldsymbol{r}_{i}\right|\right)+\frac{D_{s}}{n_{0}} \sum_{j \neq i} \frac{\left(p_{i}-\hat{p}_{i}\right)}{\left|\boldsymbol{r}_{j}-\boldsymbol{r}_{i}\right|^{2}}\left(\boldsymbol{r}_{j}-\boldsymbol{r}_{i}\right) w\left(\left|\boldsymbol{r}_{j}-\boldsymbol{r}_{i}\right|\right)
$$

Similarly in the CMPS method (Eq. 3):

$$
\langle\nabla p\rangle_{i}=\frac{D_{s}}{n_{0}} \sum_{j \neq i} \frac{\left(p_{j}-p_{i}\right)}{\left|\boldsymbol{r}_{j}-\boldsymbol{r}_{i}\right|^{2}}\left(\boldsymbol{r}_{j}-\boldsymbol{r}_{i}\right) w\left(\left|\boldsymbol{r}_{j}-\boldsymbol{r}_{i}\right|\right)+\frac{D_{s}}{n_{0}} \sum_{j \neq i} \frac{\left(p_{i}-\hat{p}_{i}\right)+\left(p_{i}-\hat{p}_{j}\right)}{\left|\boldsymbol{r}_{j}-\boldsymbol{r}_{i}\right|^{2}}\left(\boldsymbol{r}_{j}-\boldsymbol{r}_{i}\right) w\left(\left|\boldsymbol{r}_{j}-\boldsymbol{r}_{i}\right|\right)
$$

In the right hand of both Eqs. 4 and 5, because the first term is the mathematical definition of gradient term, the second term can be regarded as the artificial repulsive force term. Focusing on pressure differences in the numerators, we can write:

$$
\left|\left(p_{i}-\hat{p}_{i}\right)+\left(p_{i}-\hat{p}_{j}\right)\right| \geq\left\{\begin{array}{l}
\left|p_{i}-\hat{p}_{i}\right| \mid \\
\{\text { or } \\
\left\{\left.\left|p_{i}-\hat{p}_{j}\right|\right|_{j}\right.
\end{array}\right\} \geq\left|p_{j}-p_{i}\right|
$$

Therefore, the stability with regard to Eqs. 1 and 3 would depend on pressure states and particle distributions. And the artificial repulsive force is clearly predominant in these equations rather than the original gradient. On that account, the gradient operators may result in overestimation of interparticle pressure forces and, as a result, would bring about unphysical fluid motions and perturbations.

Here we propose a Dynamically Stabilized (DS) gradient operator comprising of the original Taylor-series consistent gradient model [1] and a meticulously adequate stabilizing force. 


$$
\begin{aligned}
& \langle\nabla p\rangle_{i}=\frac{D_{s}}{n_{0}} \sum_{j \neq i} \frac{\left(p_{j}-p_{i}\right)}{\left|\boldsymbol{r}_{j}-\boldsymbol{r}_{i}\right|^{2}}\left(\boldsymbol{r}_{j}-\boldsymbol{r}_{i}\right) w\left(\left|\boldsymbol{r}_{j}-\boldsymbol{r}_{i}\right|\right)+\frac{1}{n_{0}} \sum_{j \neq i} \boldsymbol{F}_{i j}^{D S} w\left(\left|\boldsymbol{r}_{j}-\boldsymbol{r}_{i}\right|\right) \\
& \left\{\begin{array}{l}
\boldsymbol{F}_{i j}^{D S}=0 \quad \text { if }\left|\boldsymbol{r}_{j i}^{*}\right| \geq d_{j i} \quad ; \quad d_{i j}=\alpha_{D S} \frac{d_{i}+d_{j}}{2}, \quad \Pi_{i j}>0 \\
\boldsymbol{F}_{i j}^{D S}=-\rho_{i} \Pi_{i j} \boldsymbol{e}_{j i, / /} \quad \text { if }\left|\boldsymbol{r}_{j i}^{*}\right|<d_{j i}
\end{array}\right.
\end{aligned}
$$

where $\boldsymbol{F}_{i j}{ }^{D S}$ is the stabilizing force for target particle $i$ from neighboring particle $j, \rho$ is particle density, $\Pi_{i j}$ is parameter to adjust the magnitude of $\boldsymbol{F}_{i j}{ }^{D S}, \boldsymbol{r}_{j i}=\boldsymbol{r}_{j}-\boldsymbol{r}_{i}, \boldsymbol{e}_{j i, / /}$ is the unit vector of $\boldsymbol{r}_{j i}, d$ is particle diameter, and $\alpha_{D S}$ is constant for adjusting active range of $\boldsymbol{F}_{i j}{ }^{D S}$. The superscript $*$ refers to a state after the advection by the original gradient term. $\alpha_{D S}$ is decided according to Courant stability condition for a time resolution as:

$\alpha_{D S}+\alpha_{d t}=1$

where $\alpha_{d t}$ is the ratio of the time step to Courant number $(=0.1)$ [13]. In fact, Dynamic Stabilization is activated if a neighboring particle $j$ overlaps into the target particle $i$ by more than $10 \%$ of the initial spacing. The intermediate position vector $\boldsymbol{r}_{j i}^{*}$ can be expressed with the parallel and normal vectors as:

$$
\boldsymbol{r}_{j i}^{*}=\boldsymbol{r}_{j i, / /}^{*}+\boldsymbol{r}_{j i, \perp}^{*}
$$

where $\boldsymbol{r}_{j i, /}^{*}$ is the parallel vector of $\boldsymbol{r}_{j i}^{*}$, and $\boldsymbol{r}_{j i, \perp}^{*}$ is normal vector of $\boldsymbol{r}_{j i}^{*}$. The parallel and normal vectors in the above equation can be obtained from the following equations:

$$
\left\{\begin{array}{l}
\boldsymbol{r}_{j i, / /}^{*}=\boldsymbol{r}_{j i}+\frac{\Delta \boldsymbol{r}_{j i}^{*} \cdot \boldsymbol{r}_{j i}}{\left|\boldsymbol{r}_{j i}\right|} \boldsymbol{e}_{j i, / /} \\
\boldsymbol{r}_{j i, \perp}^{*}=\frac{\left|\Delta \boldsymbol{r}_{j i}^{*} \times \boldsymbol{r}_{j i}\right|}{\left|\boldsymbol{r}_{j i}\right|} \boldsymbol{e}_{j i, \perp}
\end{array} ; \Delta \boldsymbol{r}_{j i}^{*}=-\left(\frac{\langle\nabla p\rangle_{j}}{\rho_{j}}-\frac{\langle\nabla p\rangle_{i}}{\rho_{i}}\right) \Delta t^{2}\right.
$$

where $\boldsymbol{e}_{j i, \perp}$ is the unit vector of $\boldsymbol{r}_{j i, \perp}$. As shown in Fig. 1, when particles overlap with each other, the stabilizing repulsive force is given to make them separated and verged on each other as the least, that is the adequate repulsive force. The stabilizing forces between particles $i$ and $j$ act at the opposite directions along the $\boldsymbol{e}_{j i, / /}$ respectively to satisfy anti-symmetric relation in accordance with the Newton's third law, that is:

$$
\boldsymbol{F}_{i j}=-\boldsymbol{F}_{j i} \quad ; \quad \boldsymbol{F}_{i j}=\boldsymbol{F}_{i j, / /}, \quad \boldsymbol{F}_{j i}=\boldsymbol{F}_{j i, / /}
$$


Stabilizing force is based on the instantaneous distribution of particles without any pressure value as:

$$
\left|\boldsymbol{r}_{j i}^{*}\right|=\left|\boldsymbol{r}_{j i, / /}^{*}+\boldsymbol{r}_{j i, \perp}^{*}\right|<d_{j i}=\left|\boldsymbol{r}_{j i}^{D S}\right|=\left|\boldsymbol{r}_{j i, / /}^{*}+\boldsymbol{r}_{j i, \perp}^{*}+\Delta \boldsymbol{r}_{j i}^{D S}\right|
$$

where $\boldsymbol{r}_{i j}{ }^{D S}$ is the coordinate vector through revision. From Eq. 13, the following equations can be obtained:

$$
\begin{aligned}
& \sqrt{d_{j i}^{2}-\left|\boldsymbol{r}_{j i, \perp}^{*}\right|^{2}}=\left|\boldsymbol{r}_{j i, / /}^{*}+\Delta \boldsymbol{r}_{j i}^{D S}\right| \\
& \left|\boldsymbol{r}_{j i, / /}^{*}\right|<\left|\boldsymbol{r}_{j i, / /}^{*}+\Delta \boldsymbol{r}_{j i}^{D S}\right|
\end{aligned}
$$

Therefore,

$$
\left|\boldsymbol{r}_{j i, / /}^{*}+\Delta \boldsymbol{r}_{j i}^{D S}\right|=\left|\boldsymbol{r}_{j i, / /}^{*}\right|+\left|\Delta \boldsymbol{r}_{j i}^{D S}\right|
$$

Considering Eq. 12, revising coordinate vector $\Delta \boldsymbol{r}_{i j}{ }^{D S}$ is derived from Eq. 8:

$$
\begin{aligned}
\Delta \boldsymbol{r}_{j i}^{D S}=\Delta \boldsymbol{u}_{j i}^{D S} \Delta t=\Delta \boldsymbol{a}_{j i}^{D S} \Delta t^{2} & =\left(\frac{\boldsymbol{F}_{j i}^{D S}}{\rho_{j}}-\frac{\boldsymbol{F}_{i j}^{D S}}{\rho_{i}}\right) \Delta t^{2}=\left(-\frac{\boldsymbol{F}_{i j}^{D S}}{\rho_{j}}-\frac{\boldsymbol{F}_{i j}^{D S}}{\rho_{i}}\right) \Delta t^{2} \\
& =\left(\frac{\rho_{i} \Pi_{i j}}{\rho_{j}}+\frac{\rho_{i} \Pi_{i j}}{\rho_{i}}\right) \boldsymbol{e}_{j i} \Delta t^{2}=\Pi_{i j}\left(1+\frac{\rho_{i}}{\rho_{j}}\right) \boldsymbol{e}_{j i} \Delta t^{2}
\end{aligned}
$$

From Eqs. 14, 16, and 17:

$$
\begin{aligned}
\sqrt{d_{j i}^{2}-\left|\boldsymbol{r}_{j i, \perp}^{*}\right|^{2}}-\left|\boldsymbol{r}_{j i, / /}^{*}\right| & =\left|\Delta \boldsymbol{r}_{j i}^{D S}\right| \\
& =\left|\Pi_{i j}\left(1+\frac{\rho_{i}}{\rho_{j}}\right) \boldsymbol{e}_{j i} \Delta t^{2}\right|=\Pi_{i j}\left(1+\frac{\rho_{i}}{\rho_{j}}\right) \Delta t^{2}
\end{aligned}
$$

Therefore,

$$
\Pi_{i j}=\frac{\rho_{j}}{\Delta t^{2}\left(\rho_{i}+\rho_{j}\right)}\left(\sqrt{d_{j i}^{2}-\left|\boldsymbol{r}_{j i, \perp}^{*}\right|^{2}}-\left|\boldsymbol{r}_{j i, / /}^{*}\right|\right)
$$

The adequate stabilizing forces $\boldsymbol{F}_{i j}{ }^{D S}$ are calculated by the prediction of the particle positions based on the original gradient term corresponding to the first term in the right hand side of Eq. 7. Firstly, the predicted relative distance between particles $i$ and $j$ is obtained from Eq. 11. If the particles would penetrate each other in the prediction, parameter $\Pi_{i j}$ to adjust the magnitude of $\boldsymbol{F}_{i j}{ }^{D S}$ is calculated by Eq. 19. In this process, $\boldsymbol{F}_{i j}{ }^{D S}$ is considered on the basis of a one-to-one relative position of a target particle $i$ and a neighboring particle $j$ and thus, the pressure gradient at particle $i$ is obtained by considering the 
distribution of all neighboring particles through a weighted averaging of $\boldsymbol{F}_{i j}{ }^{D S}$ as shown in Eq. 7.

\section{Numerical simulation}

\subsection{Evolution of a square patch of fluid [14]}

To test the stability of the proposed scheme in tensile stress states, the evolution of a $2 \mathrm{D}$ square patch of water subjected to a rigid rotation is performed. The evolution of the fluid patch is governed by the continuity and Euler equations [14]. In the present simulation, as an initial condition, the square patch is set as $1.0 \mathrm{~m}$ in length $(L=1.0 \mathrm{~m})$ comprising of water particles with $d=2.0 \times 10^{-2} \mathrm{~m}$. The angular velocity is set as $\omega=1.0 \mathrm{~s}^{-1}$.

Fig. 2 shows snapshots of water particles and pressure field by MPS-HS-GC and MPS-HS-GC-DS methods. From Fig. 2(a), the MPS-HS-GC snapshot at $t=0.84 \mathrm{~s}$ is characterized by an unphysical pressure field and dispersed particles in the vicinity of the patch boundaries. The MPS-HS-GC simulation breaks up at this moment. On the other hand, in Fig. 2b, the MPS-HS-GC-DS snapshot shows a stable state without unphysical particle clustering and fragmentation. The MPS-HS-GC-DS simulation continues to run and maintains its stable state tenaciously (shown in Fig. 2c and d). Hence, the Dynamically Stabilized scheme provides a fully stable simulation of a highly deformed free-surface flow characterized by a tensile stress state.

\subsection{Numerical simulation of two-phase flows}

In this section, the stability and performance of the proposed scheme in reproduction of multi-phase flows are examined through some benchmark numerical tests. For comparison, these numerical tests are implemented by three numerical models listed as follows.

1. CMPS-HS: An improved particle method using the CMPS gradient scheme [11] and a Higher order Source of Poisson pressure equation [12], which is effective in enhancing the numerical stability (and can treat only repulsive interaction between particles).

2. MPS-HS-GC: An enhanced particle method utilizing the HS scheme [12] and a Gradient Correction (GC) [8], which is effective in improvement of accuracy (and can treat both repulsive and tensile interactions). 
3. MPS-HS-GC-DS: An enhanced particle method using the HS [12], GC [8] and the newly DS scheme.

\subsubsection{Settlement of heavier fluid in water}

Numerical simulation of a simple typical two-phase flow, i.e. settlement of heavier fluid particles in water, is performed with CMPS-HS, MPS-HS-GC and MPS-HS-GC-DS methods. The initial condition is set as shown in Fig 3. The tank is $0.25 \mathrm{~m}$ in length and $0.2 \mathrm{~m}$ in height, and is filled with 7000 fluid particles with $d=2.5 \times 10^{-3} \mathrm{~m}$, resulting in a water depth of $0.175 \mathrm{~m}$. The densities of the lighter particles $\rho_{L}$ and the heavier particles $\rho_{H}$ are set as $\rho_{L}=1000.0 \mathrm{~kg} / \mathrm{m}^{3}$ and $\rho_{H}=2650.0 \mathrm{~kg} / \mathrm{m}^{3}$, respectively. From Fig 4, the CMPS-HS method reproduces a stable free-surface, however, vectors of velocity are unphysical with perturbations. In addition, some heavier particles do not settle down and are fully bounded within the lighter fluid particles. The cause of this unphysical result is likely brought about by a too strong stabilizing force (Eq. 5). The results by MPS-HS-GC are characterized by an unstable free-surface, in particular, at $t=0.4 \mathrm{~s}$. This unphysical reproduction leads to numerical instability at $t=0.41 \mathrm{~s}$. On the other hand, MPS-HS-GC-DS portrays a physically sound reproduction of the phenomenon. The snapshots by this method show a smooth free-surface with reproducing the circulating flow driven by the settlement of heavier fluid. The settlement of heavier fluid particles is well simulated by this method.

\subsubsection{Surfacing of lighter fluid in water}

Simulation of a two-phase flow with surfacing of lighter fluid particles in water is performed (Fig. 5) with CMPS-HS, MPS-HS-GC and MPS-HS-GC-DS methods. The initial set up of calculation corresponds to that in Section 3.2.1, except for replacing a fluid lighter than water with $\rho_{L}=600.0 \mathrm{~kg} / \mathrm{m}^{3}$ in place of the previously heavier fluid. As shown in Fig. 6, in spite of the smooth free-surface line, CMPS-HS recurrently shows unphysical perturbation of velocity even in the static state at $t=10.00 \mathrm{~s}$. Further, lighter particles stay in water, or are bounded within heavier (water) particles. While, MPS-HS-GC reproduces rather smoother velocity field, surface particles behave unstably and the simulation breaks up at $t=0.35 \mathrm{~s}$. The MPS-HS-GC-DS shows a good reproduction in both velocity field (circulating flow driven by surfacing of particles) and behavior of particles. This scheme resolves all problems shown in the results of the other schemes. 


\section{Concluding remarks}

A novel scheme is proposed for Dynamic Stabilization of a particle method, namely, the MPS method. The new scheme, abbreviated as DS, provides precisely adequate repulsive interparticle forces based on the instantaneous distribution of particles to eliminate the interparticle penetration which is the main cause of instability in particle methods. A few numerical tests, namely, evolution of a square patch of fluid and a set of simple two-phase flow simulations have been performed to show the stabilizing and enhancing effects of the DS scheme. Despite the fact that this paper focuses on Dynamic Stabilization of 2D MPS-based simulations, the major finding and developments can be easily applied and extended to 3D simulations as well as other particle methods, i.e. the SPH method.

\section{Acknowledgment}

This research was supported by Grant-in-Aid for JSPS Fellows from Japan Society for the Promotion of Science (No. 24-4720).

\section{References:}

[1] S. Koshizuka and Y. Oka, "Moving particle semi-implicit method for fragmentation of incompressible fluid," Nuclear Science and Engineering, 123, 421-434, 1996.

[2] J.W. Swegle, D. L. Hicks and S. W. Attaway, "Smoothed Particle Hydrodynamics Stability Analysis", Journal of Computational Physics, 116, 123, 1995.

[3] J.K. Chen, J.E. Beraun and C.J. Jih, "An improvement for tensile instability in smoothed particle hydrodynamics”, Comput. Mech., 23, 279-287, 1999.

[4] J.J. Monaghan, "SPH without a tensile instability", Journal of Computational Physics, 159(2), 290-311, 2000.

[5] J. Bonet and S. Kulasegaram, "Remarks on tension instability of Eulerian and Lagrangian corrected smooth particle hydrodynamics (CSPH) methods", International Journal of Numerical Methods in Engineering, 52, 1203-1220, 2001. 
[6] L.D.G. Sigalotti and H. Lopez, "Adaptive kernel estimation and SPH tensile instability", Computers and Mathematics with Applications, 55(1), 23-50, 2008.

[7] Y. Meleán, L. Di, G. Sigalotti and A. Hasmy, "On the SPH tensile instability in forming viscous liquid drops", Computer Physics Communications, 157, 191, 2004.

[8] A. Khayyer and H. Gotoh, "Enhancement of stability and accuracy of the moving particle semi-implicit method", Journal of Computational Physics, 230, 3093-3118, 2011.

[9] A. Khayyer and H. Gotoh, "Enhancement of performance and stability of MPS meshfree particle method for multiphase flows characterized by high density ratios", Journal of Computational Physics, 242, 211-233, 2013.

[10] S. Koshizuka, A. Nobe and Y. Oka, "Numerical Analysis of Breaking Waves Using the Moving Particle Semi-implicit Method", International Journal for Numerical Methods in Fluids, 26, 751-769, 1998.

[11] A. Khayyer and H. Gotoh, "Development of CMPS method for accurate water-surface tracking in breaking waves,” Coast. Eng. J., 50(2), 179-207, 2008.

[12] A. Khayyer and H. Gotoh, "Modified Moving Particle Semi-implicit methods for the prediction of 2D wave impact pressure", Coastal Engineering, 56, 419-440, 2009.

[13] A. Khayyer, H. Gotoh and S.D. Shao, "Corrected Incompressible SPH method for accurate water-surface tracking in breaking waves," Coastal Engineering, 55(3), 236-250, 2008.

[14] A. Colagrossi, "A meshless Lagrangian method for free-surface and interface flows with fragmentation”, PhD Thesis, Universita di Roma, La Sapienza, 2003. 


\section{Figure captions}

Fig. 1. A graphical presentation of the concept of the Dynamic Stabilization scheme

Fig. 2. Snapshots of water particles together with pressure field by the MPS-HS-GC and the MPS-HS-GC-DS methods in simulation of an initially square patch of fluid

Fig. 3. Initial condition of fluid particles in simulation of a two-phase flow for settlement of heavier fluid.

Fig. 4. Snapshots of fluid particles together with velocity vectors by the MPS-HS, CMPS-HS and the MPS-HS-GC-DS methods in simulation of a two-phase flow for settlement of heavier fluid

Fig. 5. Initial condition of fluid particles in simulation of a two-phase flow for surfacing of lighter fluid

Fig. 6. Snapshots of fluid particles together with velocity vectors by the MPS-HS, CMPS-HS and the MPS-HS-GC-DS methods in simulation of a two-phase flow for surfacing of lighter fluid 
$i, j \quad:$ the present positions

$i^{*}, j^{*}$ : the predicted positions derived from the pressure gradient force $i_{i j}^{\mathrm{DS}}, j_{i j}^{\mathrm{DS}}$ : the expected positions among $i \& j$ adjusted by the stabilizing force $F_{i j}^{\mathrm{DS}}, F_{j i}^{\mathrm{DS}}$

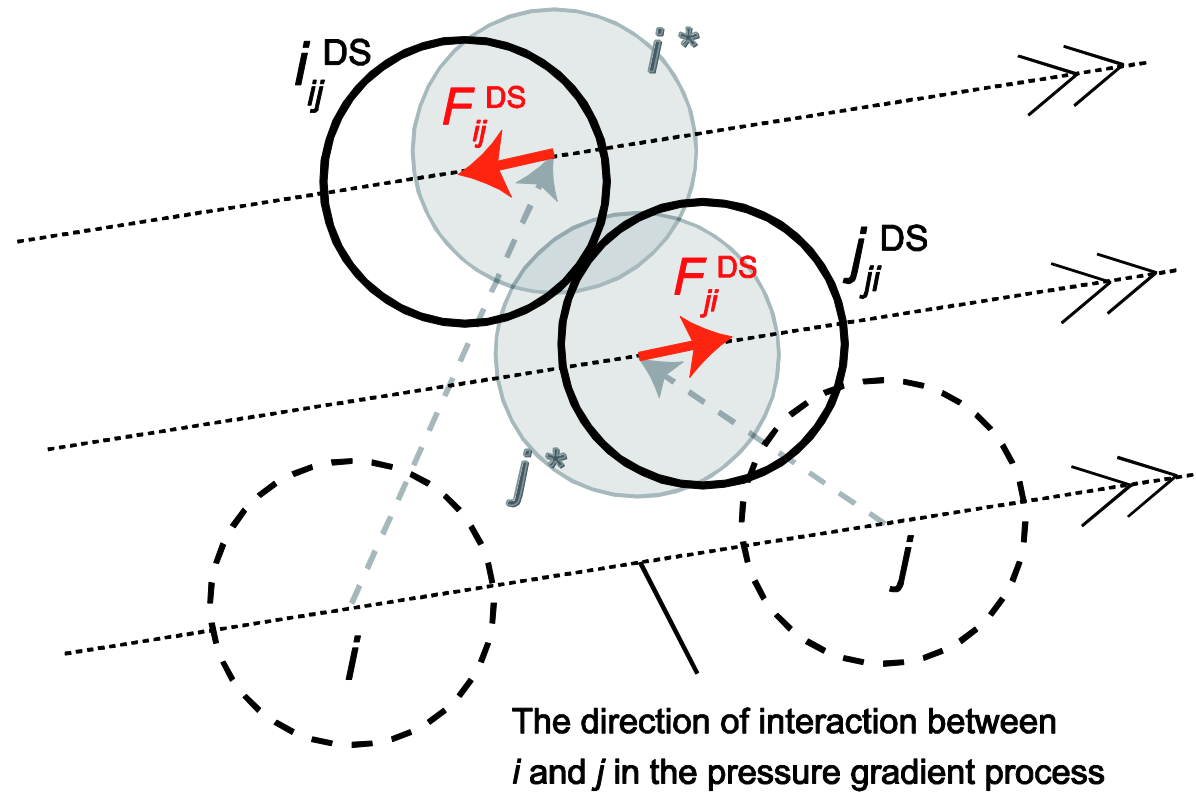

The relationship of vectors between $i$ and $j$

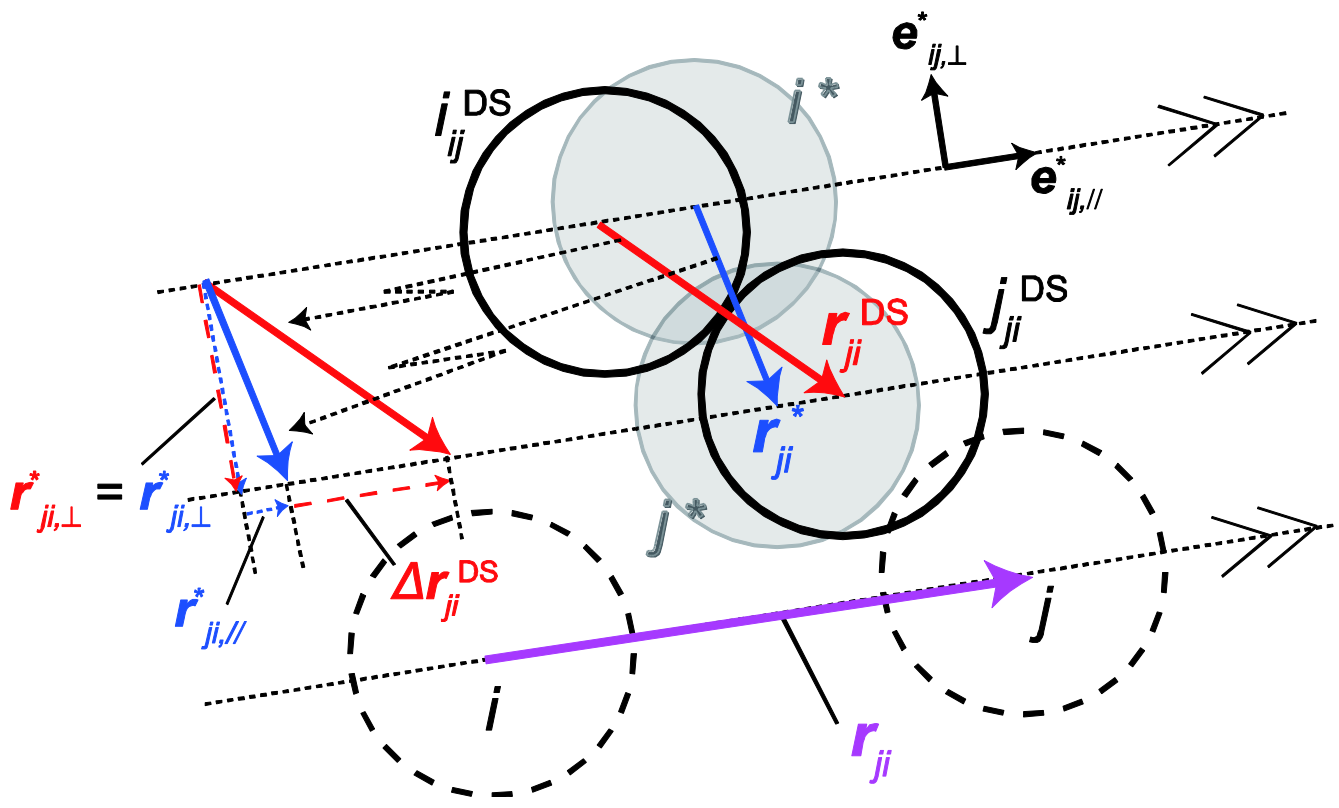

Fig. 1 

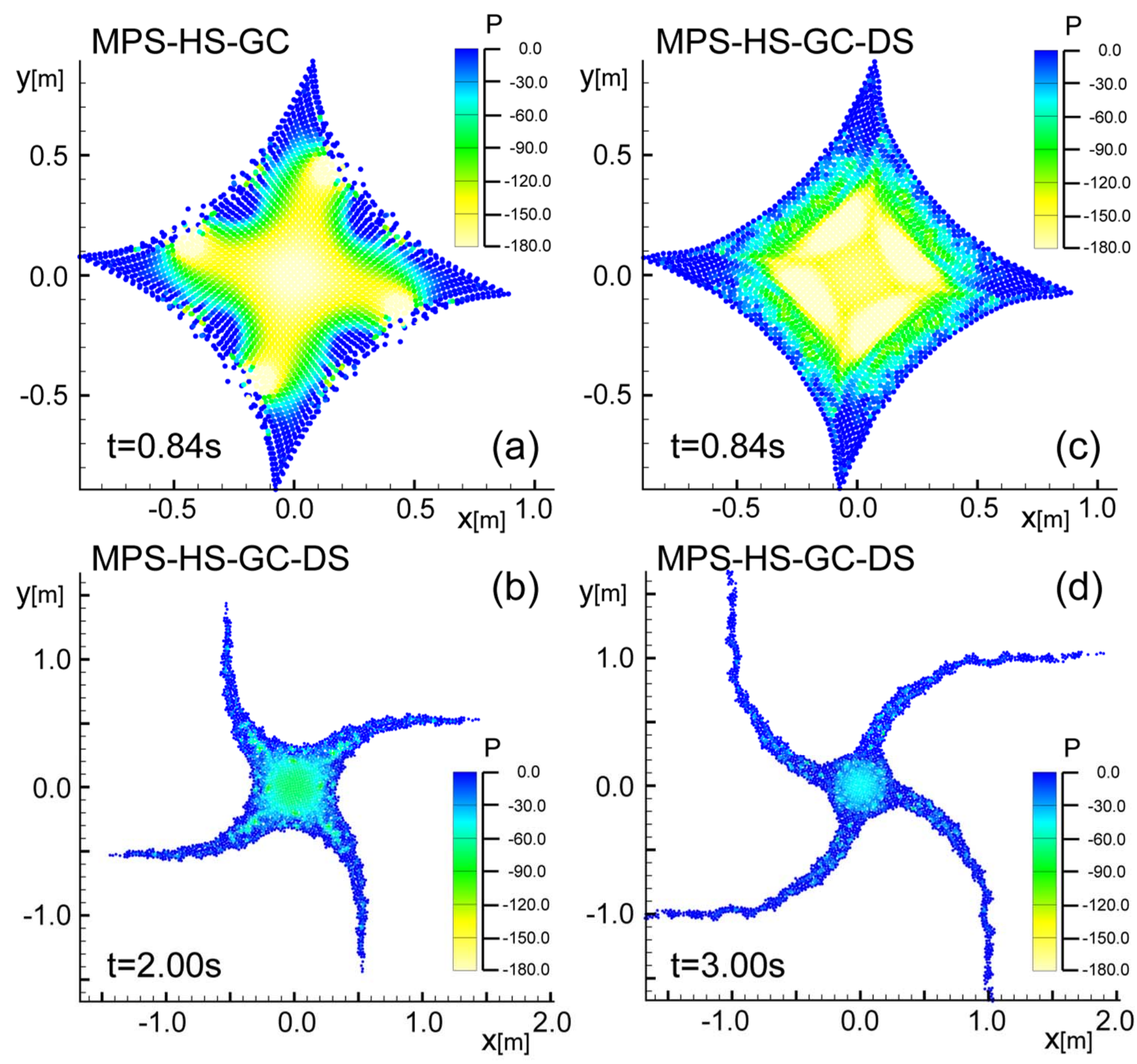

Fig. 2 


\section{Lighter particle}

\section{$\left(\rho=1000.0 \mathrm{~kg} / \mathrm{m}^{3}\right)$}

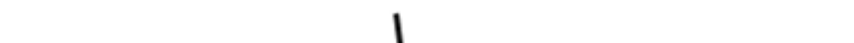

\section{Heavier particle} $\left(\rho=2650.0 \mathrm{~kg} / \mathrm{m}^{3}\right)$

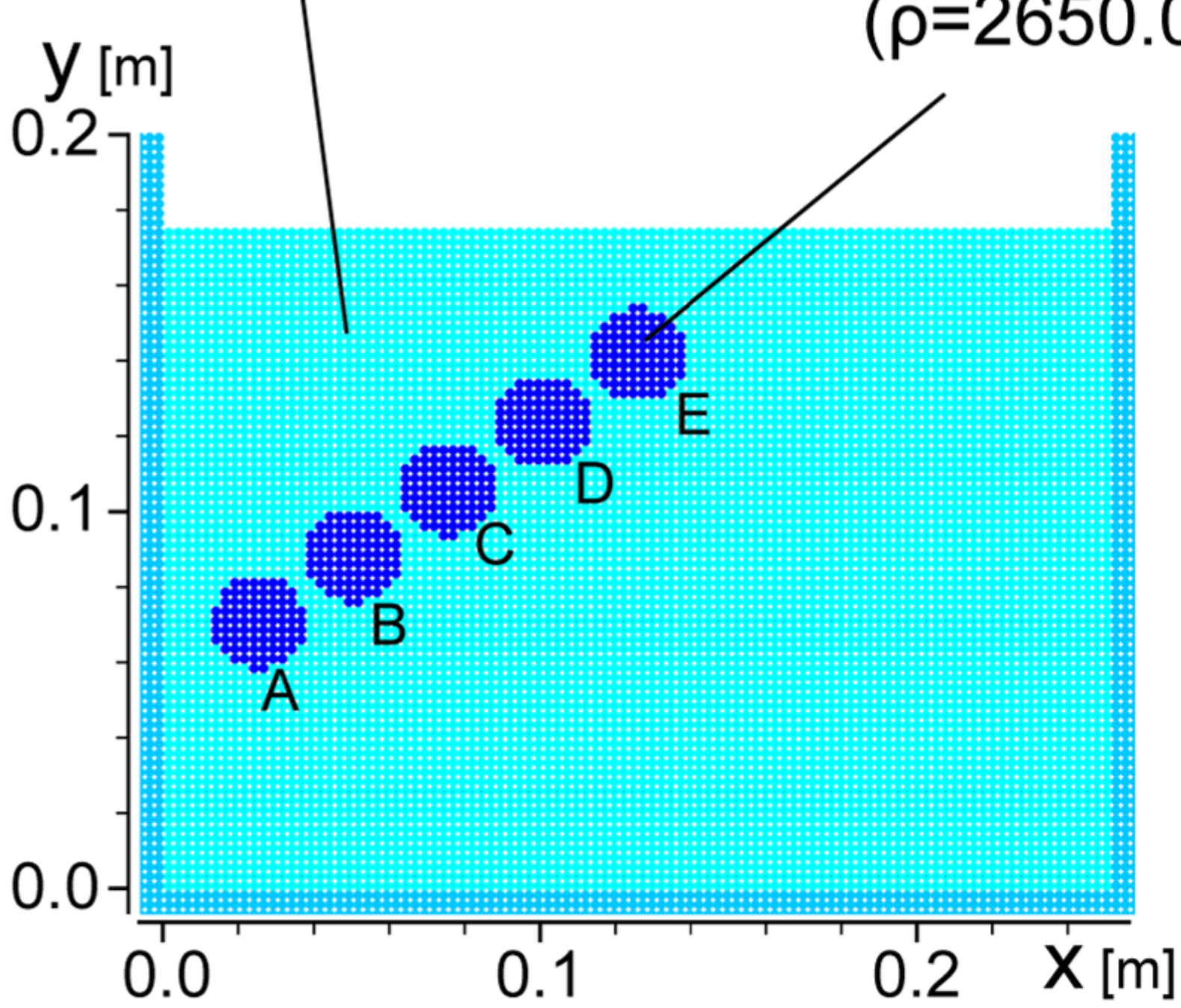

\begin{tabular}{|c|c|c|}
\hline & $\mathrm{X}[\mathrm{m}]$ & $\mathrm{Y}[\mathrm{m}]$ \\
\hline$A$ & 0.025 & 0.071 \\
\hline$B$ & 0.050 & 0.088 \\
\hline$C$ & 0.075 & 0.106 \\
\hline$D$ & 0.100 & 0.124 \\
\hline$E$ & 0.125 & 0.141 \\
\hline
\end{tabular}

Coordinates of the central point of each heavier particle group

${ }^{*}$ The diameter of each heavier particle group: $d_{\mathrm{g}}=0.025 \mathrm{~m}$ 
CMPS-HS

MPS-HS-GC

\section{MPS-HS-GC-DS}
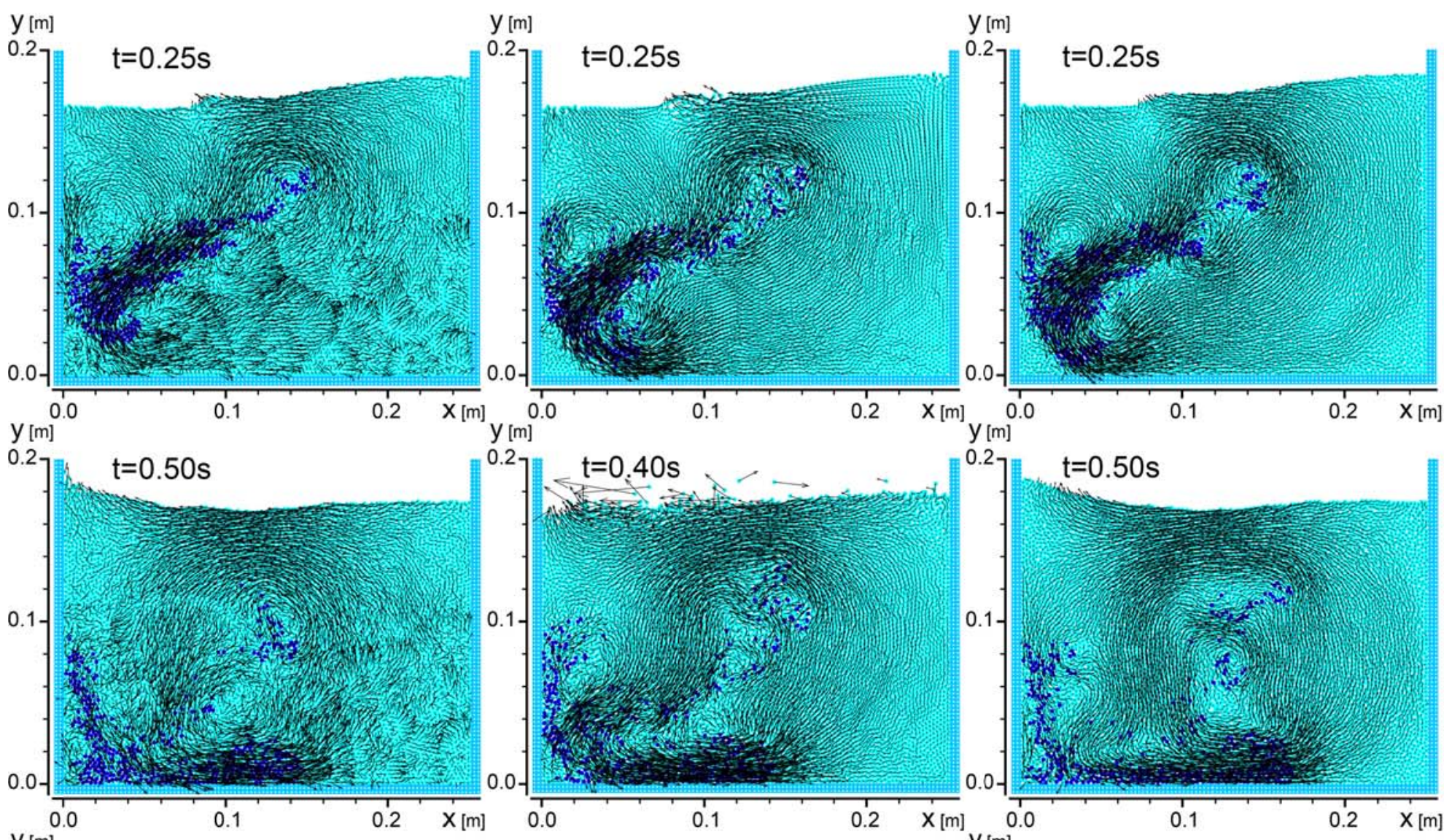

$\left.{ }^{0.2}\right] \quad t=0.50 \mathrm{~s}$
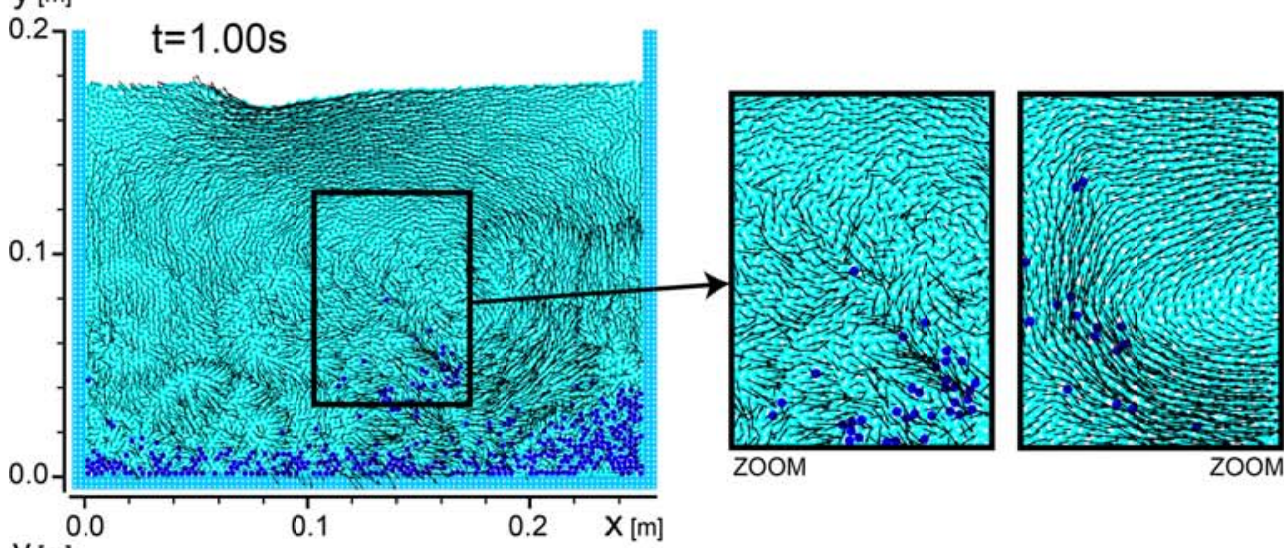

$\mathrm{y}$ [m]

$0.27 \quad t=5.00 s$
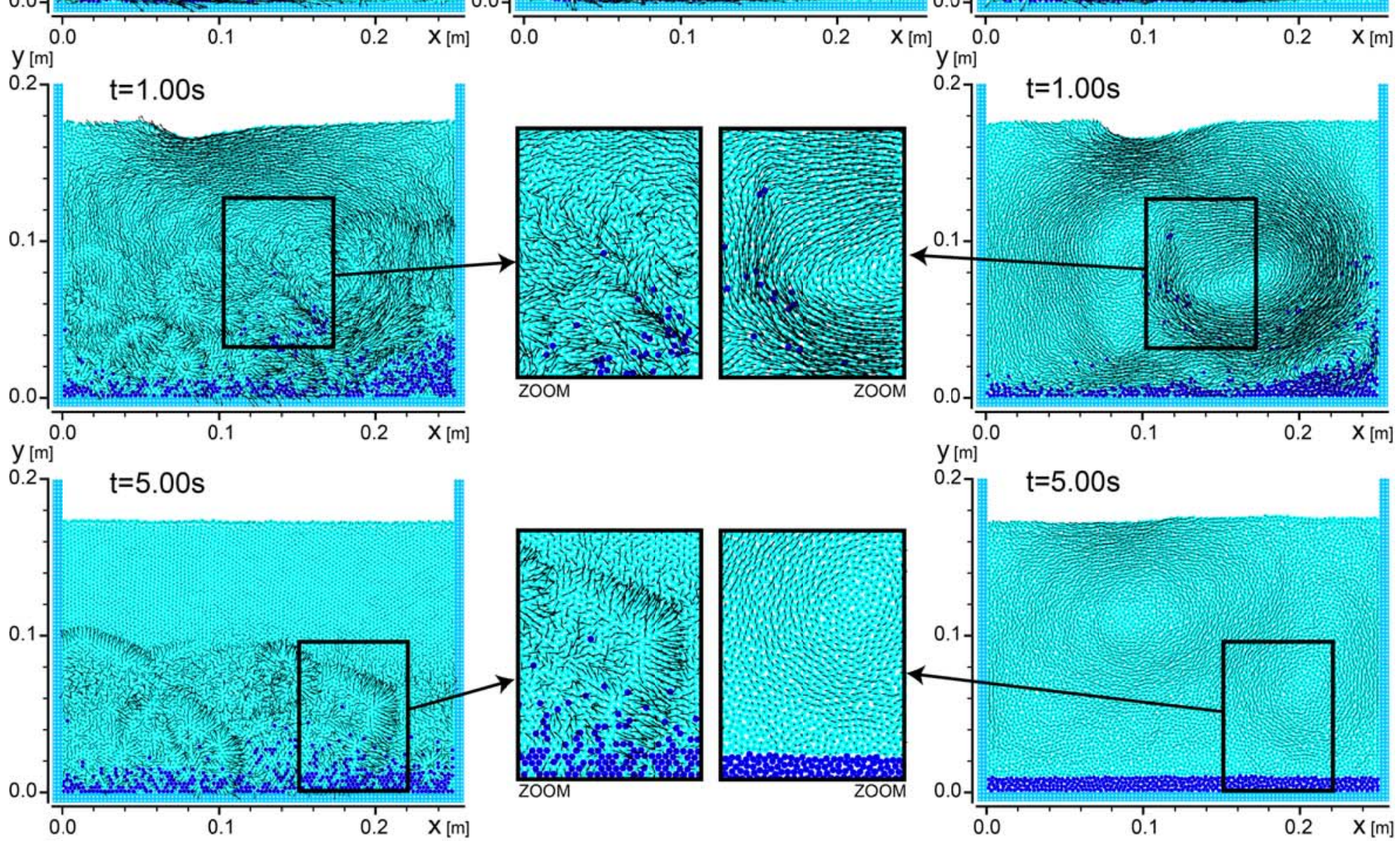

Fig. 4 


\section{Heavier particle}

$\left(\rho_{H}=1000.0 \mathrm{~kg} / \mathrm{m}^{3}\right)$

Lighter particle

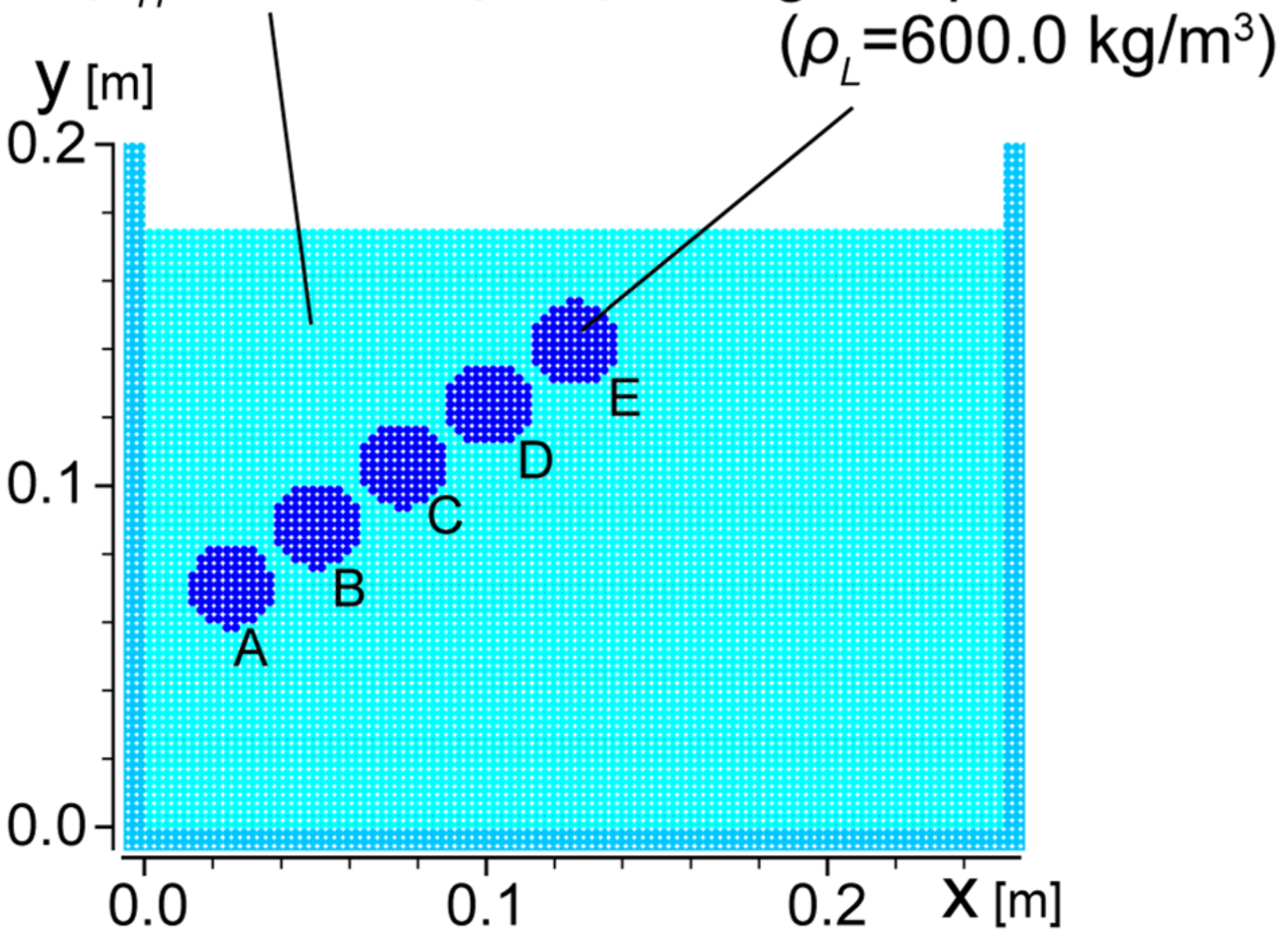

\begin{tabular}{|c|c|c|}
\hline & $\mathrm{X}[\mathrm{m}]$ & $\mathrm{Y}[\mathrm{m}]$ \\
\hline$A$ & 0.025 & 0.071 \\
\hline$B$ & 0.050 & 0.088 \\
\hline C & 0.075 & 0.106 \\
\hline$D$ & 0.100 & 0.124 \\
\hline E & 0.125 & 0.141 \\
\hline
\end{tabular}

Coordinates of the central point of each lighter particle group *The diameter of each lighter particle group: $d_{\mathrm{g}}=0.025 \mathrm{~m}$ 
CMPS-HS

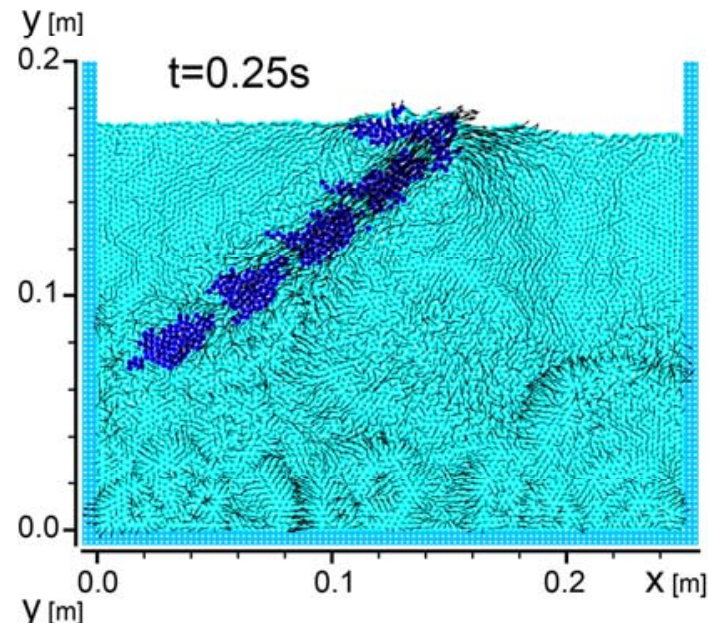

MPS-HS-GC

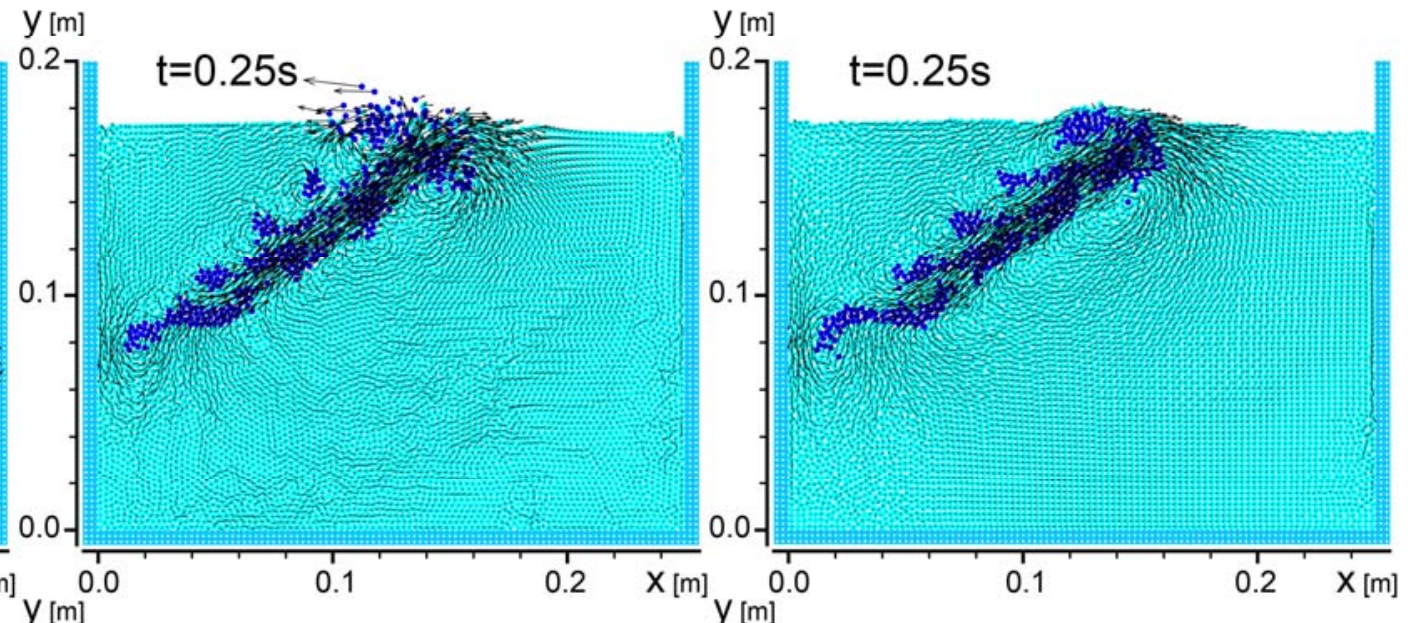

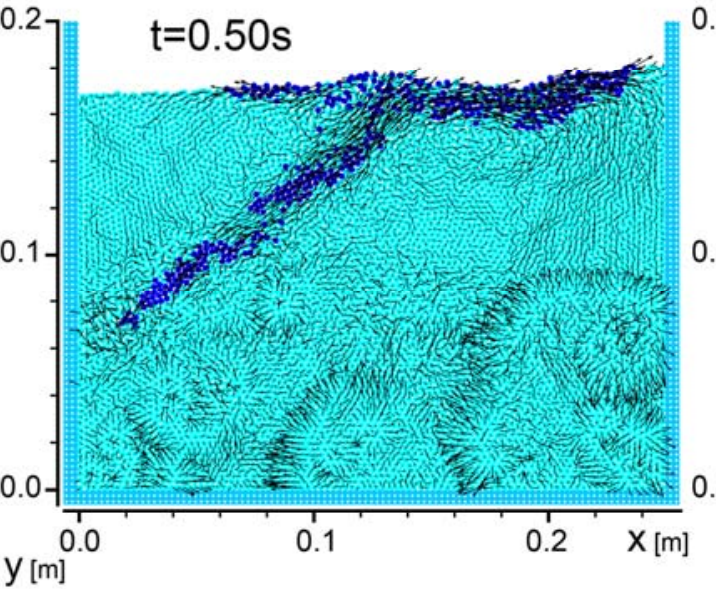

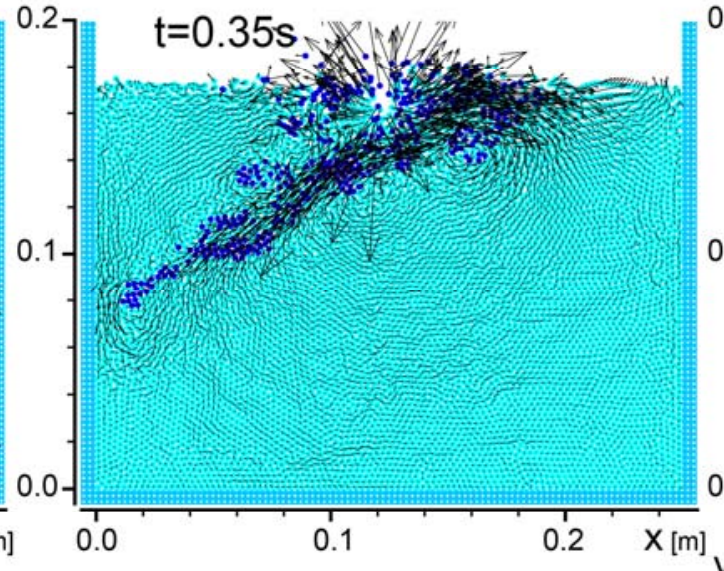

$\left.{ }^{0.2}\right] \quad t=0.50 \mathrm{~s}$
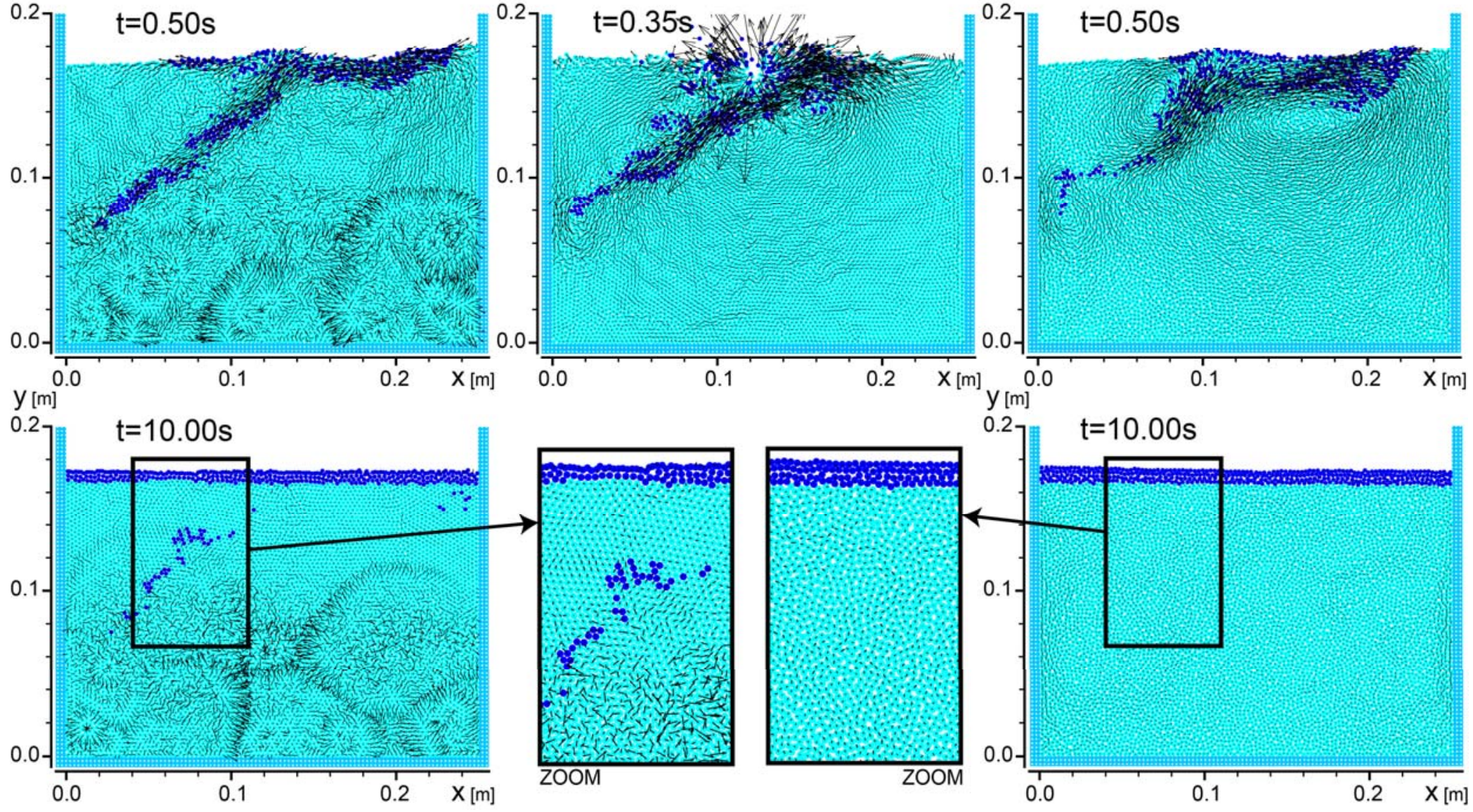

Fig. 6 